\title{
Vólvulo gástrico: ¿Por qué recordarlo? Revisión a propósito de un caso
}

Drs. Samuel Sánchez $C^{(1)}$, Laura Vique $B^{(2)}$, Oscar Ardiles $C^{(3)}$, David Herquiñigo (4). $^{(4)}$

1. Radiólogo, post-becado Hospital Clínico Universidad de Chile.

2. Becada Radiología Hospital Clínico Universidad de Chile.

3. Médico en estadía de capacitación Centro de Imagenología Hospital Clínico Universidad de Chile.

4. Radiólogo, profesor asistente Radiología Hospital Clínico Universidad de Chile.

Stomach volvulus: Why should we remember it? Case review

Abstract: Stomach volvulus is a medical entity which has different implications in terms of clinical presentation, diagnosis, imaging support, and pathological behavior and evaluation. Analysis of features of these implications is essential when deciding a course of action, which can vary from simple observation to aggressive and urgent resolutions in order to save the patient's life. Gastric volvulus represents an unusual rotation of the organ on its own axis, thus entailing risk of ischemia and necrosis. There are two major types of gastric volvulus, i.e., organoaxial and mesenteroaxial. It can occur in any stage of life, preferably in adulthood, with clinical signs of acute abdomen in most of the cases. Due to the risk of ischemia, necrosis, and vital compromise, an urgent response involves surgical resolution which can lead to the removal of the organ, with a high risk of mortality in the intra- and post-operative periods. We report the case of a patient presenting with the aforementioned clinical processes.

Keywords: Borchardt's triad, Gastrectomy, Stomach, Volvulus.

Resumen: El vólvulo gástrico es una entidad médica de diversas implicancias en cuanto a la presentación clínica, diagnóstico, apoyo imaginológico, conducta y evaluación patológica. Por tanto, es fundamental la revisión de las características de cada una de ellas, con el objeto de orientar una conducta que posee caracteres tan amplios como lo es la simple observación hasta una conducta agresiva y urgente que implique salvar la vida del paciente.

El vólvulo gástrico consiste en una rotación del órgano sobre su propio eje, de baja ocurrencia, presentándose dos tipos: organoaxial y mesenteroaxial, en los cuales existe riesgo de isquemia y necrosis. Se manifiesta en cualquier etapa de la vida, de preferencia en etapa adulta y con clínica de abdomen agudo en gran parte de los casos. Debido al riesgo de isquemia, necrosis y compromiso vital, la conducta urgente implica resolución quirúrgica, que puede concluir en extirpación del órgano, con un alto riego de mortalidad en el intra y postoperatorio.

Presentamos el caso de una paciente característica en cuanto a la presentación de los procesos clínicos antes mencionados.

Palabras clave: Estómago, Gastrectomía, Triada de Borchardt, Vólvulo.

Sánchez S, et al. Vólvulo gástrico: ¿Por qué recordarlo? Revisión a propósito de un caso. Rev Chil Radiol 2012; 18(3): 129-135.

Correspondencia: Dr. Samuel Sánchez C. / samuelsanchezc@yahoo.cl

Trabajo recibido el 06 de julio de 2012, aceptado para publicación el 02 del octubre de 2012.

Introducción

El vólvulo gástrico es una entidad clínica originada con el giro del estómago sobre su mismo eje. Este evento de rara ocurrencia, menos frecuente que en otros sitios del tracto gastrointestinal como son los vólvulos sigmoideo, cecal o de intestino medio, puede ser transitorio produciendo acaso ningún síntoma o puede llevar a una obstrucción con isquemia y necrosis.

El $75-80 \%$ corresponde a pacientes adultos, en los que por lo general, la presentación clínica está asociada a factores predisponentes congénitos y adquiridos asociados. 
El vólvulo gástrico debe ser cuidadosamente considerado como causa de epigastralgia y vómitos, pues un diagnóstico errado puede significar la muerte del paciente.

La radiología provee el medio principal para el diagnóstico y posterior enfrentamiento terapéutico de esta patología, primeramente a través de la utilización de rayos $X$ en radiología simple y contrastada para dar paso posterior a la tomografía computada, que otorga una excelente herramienta de orientación anatómica para el tratamiento.

A continuación presentamos un caso clínico de vólvulo gástrico en paciente adulto sin comorbilidad relacionada conocida con su posterior correlación imagenológica, quirúrgica y anatomopatológica.

\section{Presentación del caso}

Paciente de sexo femenino de 81 años, portadora de Miocardiopatía de Takotsubo o diskinesia apical transitoria, con antecedentes quirúrgicos de colecistectomía y artroplastía total de cadera derecha, consulta en servicio de urgencia por sintomatología de dolor abdominal epigástrico, náuseas, vómitos y sensación febril dentro de las últimas 24 horas de evolución.

El examen clínico detecta un abdomen marcadamente distendido, muy sensible y con febrícula.

Se solicita tomografía computada de abdomen y pelvis con la sospecha de hernia umbilical atascada, detectándose severa dilatación gástrica con plegamiento del órgano que ocupa hasta la excavación pelviana, compatible con vólvulo gástrico órgano-axial asociado a presencia de neumatosis gástrica y abundante gas portal, con desplazamiento y compresión de las asas intestinales hacia posterior e inferior (Figuras 1, 2, 3a, 3b). Las pruebas de laboratorio evidencian importante leucocitosis y aumento de la PCR.

Es ingresada y llevada a pabellón, en donde

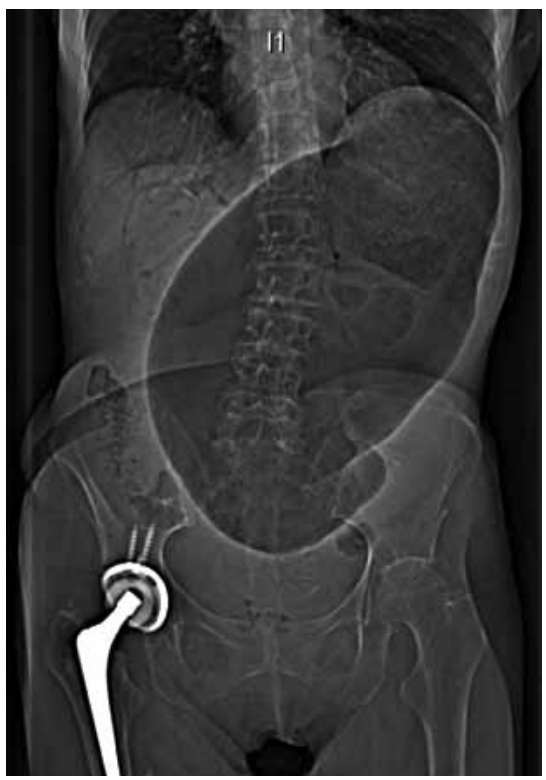

Figura 1. Scout view que muestra severa dilatación de estructura tubular que se extiende desde hipocondrio izquierdo hasta la excavación pelviana, además de signos sugerentes de aire intrahepático.

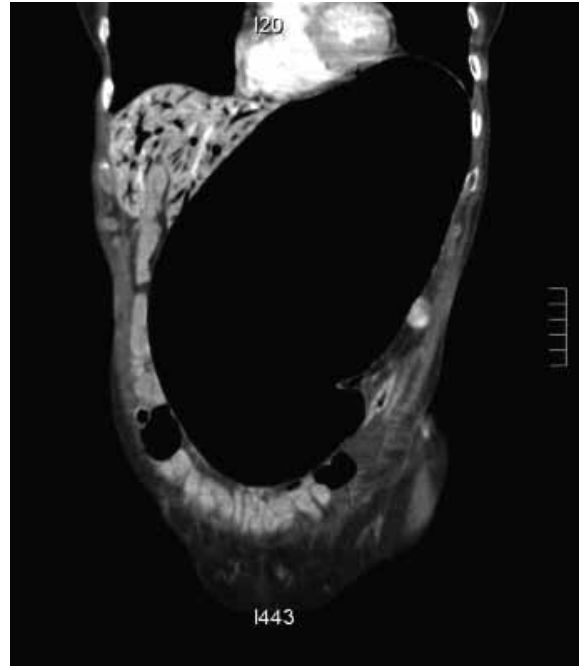

Figura 2. Reconstrucción coronal de tomografía computada que evidencia los mismos hallazgos anteriormente descritos, además de desplazamiento y compresión de asas de intestino delgado hacia la pelvis.

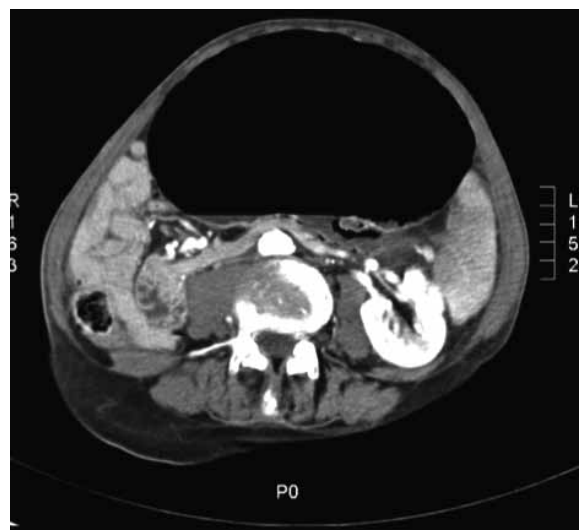

Figura 3a. Corte axial de tomografía computada que demuestra gran dilatación de estómago con signos sugerentes de neumatosis gástrica y desplazamiento de asas de intestino delgado hacia flanco derecho.

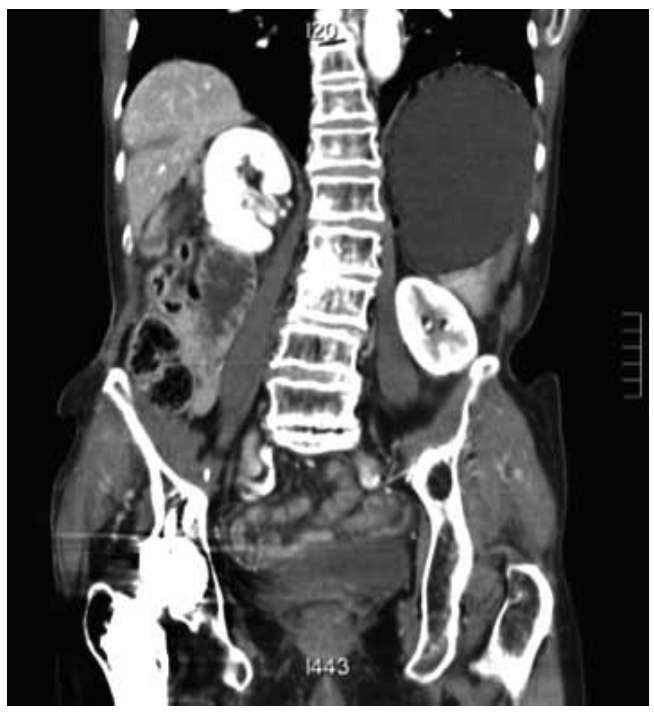

Figura 3b. Reconstrucción coronal que además presenta desplazamiento del riñón izquierdo hacia inferior. 
se realiza laparotomía exploradora amplia, encontrándose el estómago volvulado en sentido órgano-axial con evidencias de compromiso isquémico transmural predominante en cuerpo y fondo además de una perforación macroscópica, determinándose la realización de gastrectomía total y anastomosis posterior en segundo tiempo (Figuras 4, 5).

La pieza quirúrgica es llevada a anatomía patológica en donde se comprueba la presencia de pieza gástrica completa con evidencias de necrosis de cuerpo y fondo y perforación macroscópica en relación a la misma zona (Figura 6).

La paciente evoluciona satisfactoriamente desde la perspectiva médica y se programa cirugía de anastomosis en segundo tiempo.

\section{Discusión}

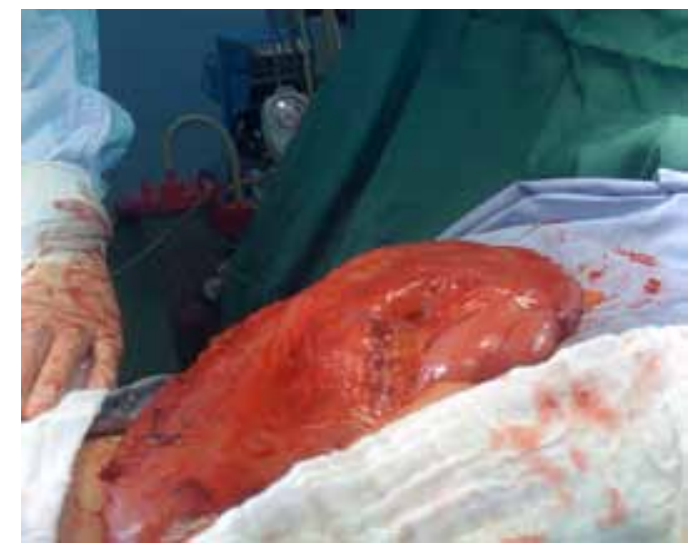

Figura 4. Imagen de estómago sobredistendido en laparotomía abierta supra e infraumbilical.

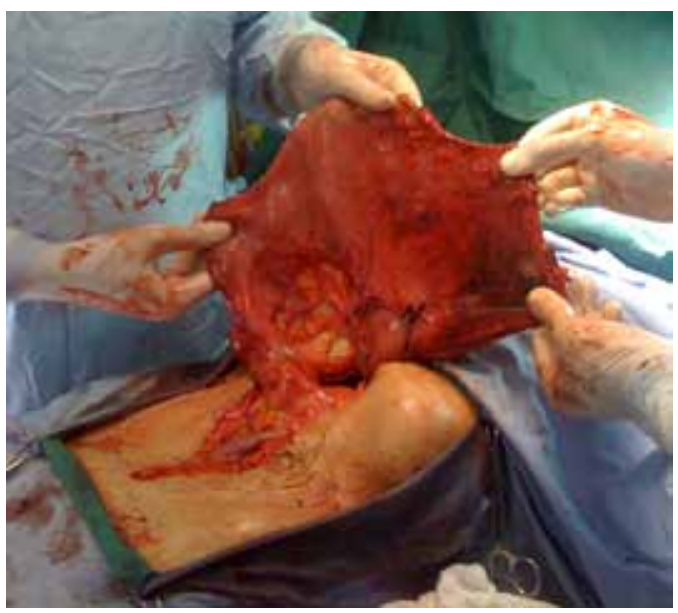

Figura 5. Imagen intraoperatoria de estómago con expresión de la mucosa con signos de isquemia y necrosis a nivel de cuerpo y fondo.

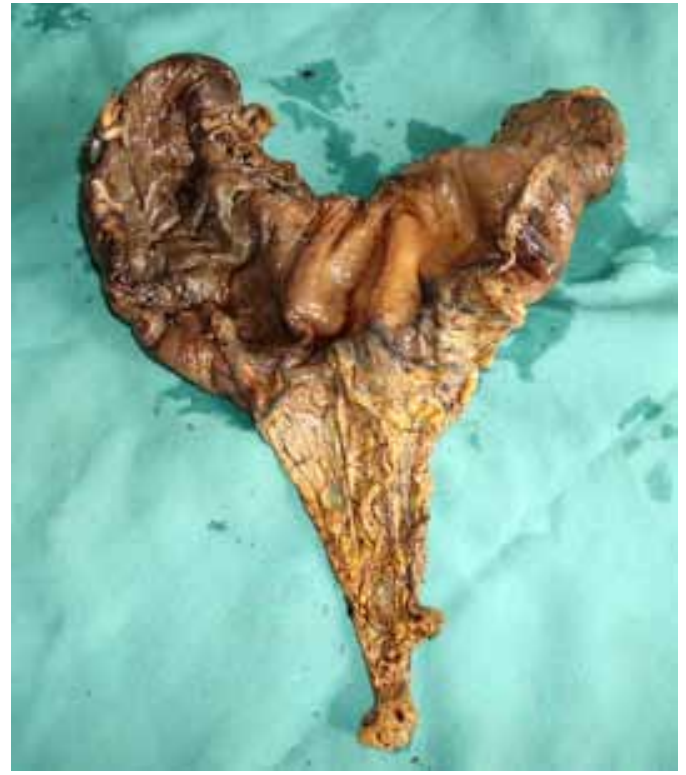

Figura 6. Pieza de anatomía patológica obtenida de gastrectomía total con signos de compromiso vascular severo a nivel de cuerpo y fondo gástrico.

El vólvulo gástrico es una entidad infrecuente. Hasta el año 2009 una publicación indicaba que se habían informado 350 casos a nivel mundial(1). Su primera descripción fue en 1866 por Berti, como un hallazgo autópsico. Posteriormente fue descrito en varias ocasiones también durante autopsias y cirugías. La primera relación radiológica fue expuesta en 1921 por Rosselet ${ }^{(2)}$.

La incidencia máxima de volvulación gástrica se sitúa en la quinta década de la vida, sin embargo, algunos autores aventuran que es mayor en niños ${ }^{(3)}$. No se encuentran diferencias significativas en su prevalencia por género o raciales, aunque algunos autores mencionan una mayor prevalencia en el sexo femenino ${ }^{(4)}$.

El vólvulo gástrico consiste en una rotación de $180^{\circ}$ del eje principal del estómago, lo cual puede producir obstrucción de la luz gástrica, asociado o no a alteración del flujo arterial. Puede resultar en complicaciones tales como isquemia gástrica, necrosis y perforación, por lo que requiere de un diagnóstico rápido.

Se han definido factores predisponentes, los cuales pueden ser divididos en idiopáticos y secundarios. Entre los factores idiopáticos destaca la elongación o ausencia de los ligamentos que fijan el estómago al peritoneo: ligamentos gastrohepático, gastrofrénico, gastrocólico y gastroesplénico. Además los estómagos anormalmente distendidos son más propensos a rotar. Otras anomalías asociadas son las hernias diafragmáticas y eventraciones del diafragma, bazo errante y mal rotación con asplenia ${ }^{(5)}$. En un estudio que analizó las autopsias de 500 pacientes con 
hernia del hiato, 12 presentaban vólvulos gástricos incompletos ${ }^{(6)}$.

Entre los factores secundarios están las operaciones gástricas reductivas como la operación de Nissen, rupturas de ligamentos gástricos tras un trasplante hepático o trauma y tumores gástricos.

Según el eje de rotación del estómago se distinguen dos tipos, el órgano-axial (58\% de los casos) y el mesenterio-axial (29\% de los casos). Se estima que alrededor del $2 \%$ de los casos corresponde a vólvulos mixtos, quedando cerca de un $10 \% \sin$ clasificar. Hasta en el $70 \%$ de los casos se asocia a defectos diafragmáticos o patología de la unión esofagogástrica (Figura 7).

El vólvulo órgano-axial, es más frecuente en adultos

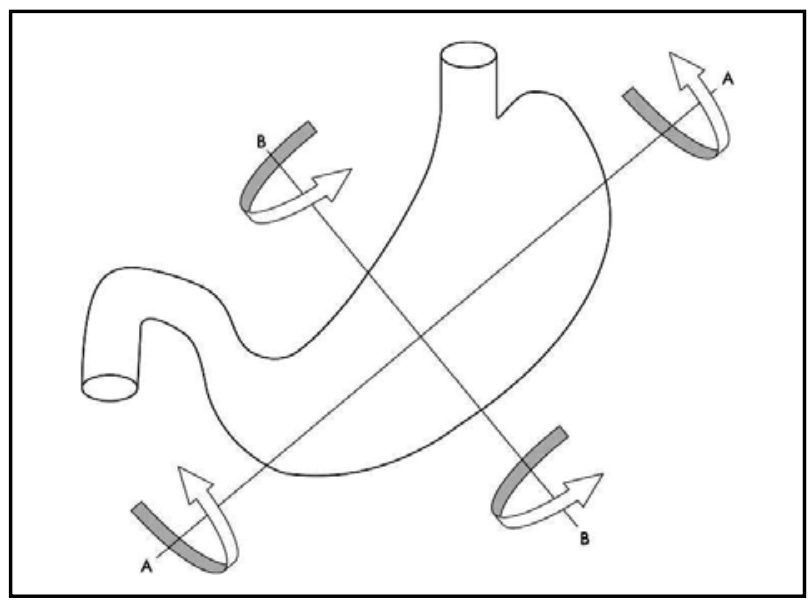

Figura 7. Esquema didáctico que distingue los 2 tipos de rotación: Organoaxial (A) y Mesenterioaxial (B).

y se asocia a estrangulación en un 5-28\%. Ocurre cuando el estómago rota sobre su propio eje o la línea que resulta al unir el píloro con la unión gastroesofágica, ubicándose la curvatura mayor hacia cefálico y la curvatura menor hacia caudal, constituyendo una urgencia quirúrgica. Es mas común en relación a trauma o hernias paraesofágicas que alteran la posición normal gástrica, pudiendo presentarse como vólvulo completo o parcial. Cuando es completo, o sea el giro es mayor o igual a 180 grados, se produce obstrucción y el estómago se dilata secundariamente. Por el contrario, si el vólvulo es parcial, es decir menor de $180^{\circ}$, puede no existir obstrucción, compromiso vascular del órgano ni sintomatología asociada. En este caso se puede hablar de que el estómago tiene una posición organoaxial y no un vólvulo organoaxial propiamente tal. Este posicionamiento del estómago predispone a desarrollar un vólvulo organoaxial, por lo que se debe realizar un seguimiento al paciente ${ }^{(7)}$.

A su vez, el vólvulo órgano-axial se puede clasificar en tipo 1 (primario) y tipo 2 (secundario). El tipo 2 es la forma de presentación más frecuente ( $2 / 3$ de los casos), su localización es supradiafragmática y suele presentarse en casos de: hernia del hiato paraesofágica, eventración diafragmática, trauma, parálisis diafragmática (lesión de nervio frénico) entre otras ${ }^{(3)}$.

El vólvulo mesenterioaxial es poco frecuente. Ocurre cuando el estómago rota sobre su eje menor, lo que resulta en el desplazamiento del antro sobre la unión gastroesofágica. Esta rotación es usualmente parcial (menor de $180^{\circ}$ ) y no se asocian a defectos diafragmáticos.

Existen además vólvulos complejos o mixtos, con componentes tanto organoaxiales como mesenterioaxiales, de baja frecuencia según lo referido en la literatura.

La presentación clínica del vólvulo gástrico puede ser aguda o crónica. El vólvulo agudo clínicamente puede presentarse con la tríada de Borchardt: dolor y distensión epigástrica, vómitos violentos o imposibilidad para vomitar y dificultad para la introducción de la sonda nasogástrica, debido a la distorsión de la anatomía en la unión gastroesofágica. Puede presentarse necrosis gástrica y tener un desenlace fatal en horas si no es resuelto mediante cirugía prontamente(5). La presentación crónica puede ser asintomática y su diagnóstico es usualmente incidental. Cursa con malestar abdominal alto que puede irradiarse al dorso u hombros, dolor durante la alimentación o post prandial temprano, saciedad precoz y vómitos. Además, en este caso se puede encontrar sintomatología de patologías frecuentemente asociadas, como hernias diafragmáticas y eventración del diafragma.

Es muy importante el rápido y oportuno diagnóstico, ya que de esto dependerá el pronóstico del paciente.

Los hallazgos radiológicos en el vólvulo mesenterioaxial, son el estómago en posición vertical con el píloro en posición cefálica al cardias y un doble nivel hidroaéreo. Además pueden encontrarse hernias diafragmáticas que pueden contener antro y píloro en su interior, constituyendo el llamado "signo del gancho"(8). En el vólvulo organoaxial el diagnóstico es más difícil: se puede observar el estómago en posición horizontal con la curvatura menor ubicada caudal a la curvatura mayor y un nivel hidroaéreo único (Figuras 8, 9). El estudio fluoroscópico con bario oral confirma el diagnóstico y el grado de obstrucción, pudiendo observarse paso del contraste en los vólvulos parciales y acumulación de éste en la zona anterior al vólvulo en las entidades completas. La tomografía computada en último término, ofrece una visión anatómica detallada del caso, además de describir las complicaciones del fenómeno entre las cuales se cuentan neumatosis gástrica, gas portal y neumoperitoneo (Figuras 10, 11). Existen descripciones aisladas en la literatura acerca de la apariencia del vólvulo organoaxial en el ultrasonido, describiéndose una doble dilatación con estrechez central, llamado el "signo del maní"(9).

El manejo del vólvulo gástrico va a depender 


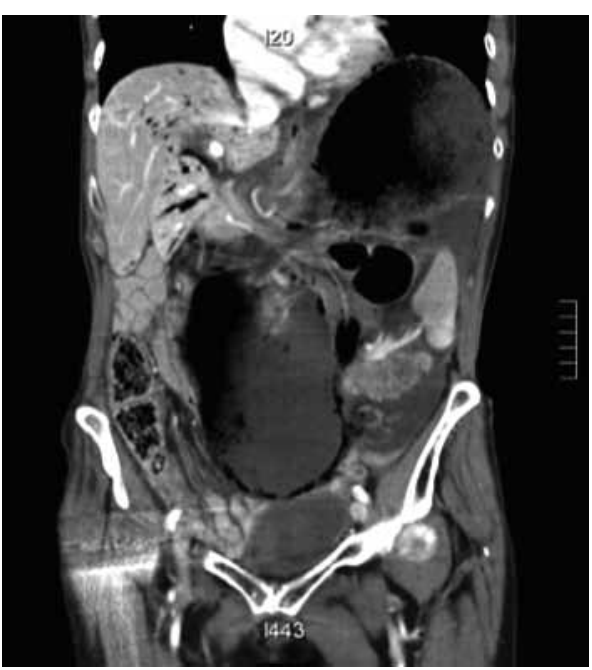

Figura 8. Reconstrucción coronal de tomografía computada que demuestra estómago severamente distendido, de distribución vertical, con un nivel hidroaéreo además de neumatosis gástrica y aire portal.

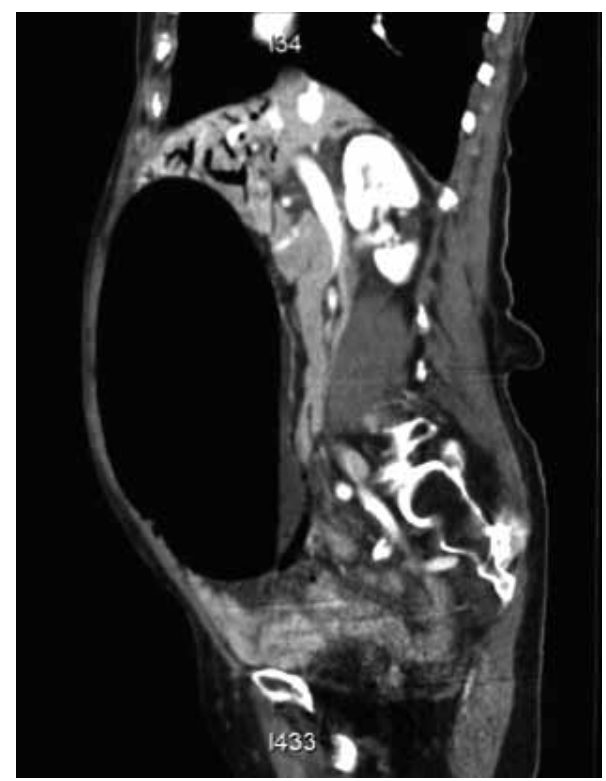

Figura 9. Reconstrucción sagital que confirma los mismos hallazgos anteriores.

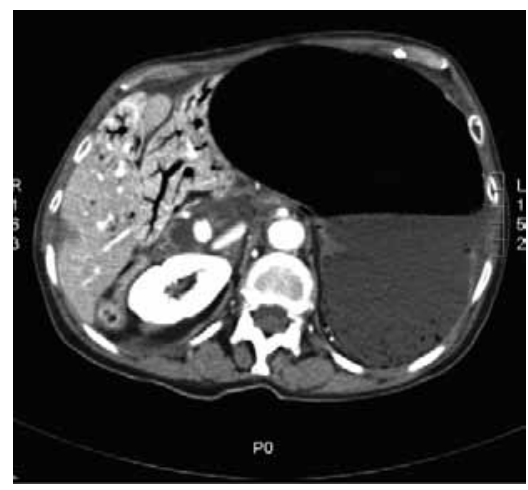

Figura 10. Corte axial de tomografía computada que presenta la severa dilatación gástrica, la compresión del parénquima hepático con aire portal y el desplazamiento a derecha de las estructuras retroperitoneales.

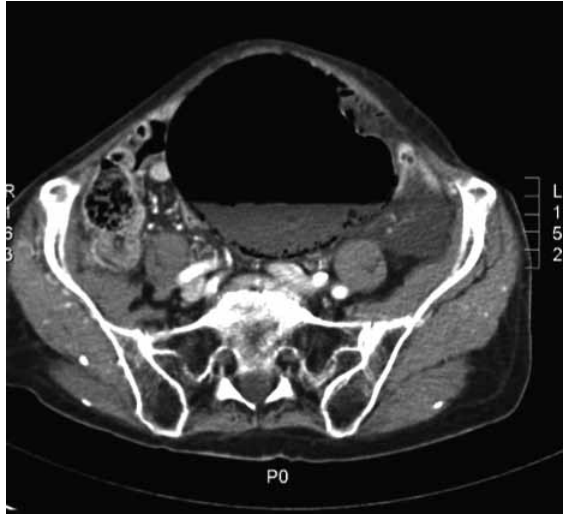

Figura 11. Corte axial de tomografía computada que muestra la presencia de estómago con neumatosis en la excavación pelviana.

principalmente de su origen y forma de presentación: aguda o crónica. Existen tres pilares en el tratamiento: reducción del vólvulo, fijación gástrica y corrección de factores predisponentes.

Se han descrito tres técnicas de enfrentamiento quirúrgico: laparotomía, laparoscopía y la vía endoscópica $^{(10)}$.

En 1968 Tanner describe varios métodos quirúrgicos para reparar el vólvulo gástrico los que incluían la reparación de hernias diafragmáticas, gastropexia, gastrectomía parcial por necrosis, gastro-gastrotomía fondo-antral (Operación de Opolzer), gastropexia con fijación del ligamento gastrocólico (Operación de Tanner), gastroyeyunostomia y reparaciones de eventraciones diafragmáticas ${ }^{(11,12)}$.

En pacientes con alto riesgo preoperatorio, la resolución endoscópica tiene buenos resultados, realizando una desvolvulación con gastrostomía percutánea por vía endoscópica ${ }^{(13)}$. Se han descrito dos técnicas para desvolvular: la maniobra Alfa-loop, descrita por Tsang y cols en 1995, la que comprende 6 pasos que buscan inicialmente formar un asa en alfa en el extremo proximal del estómago volvulado, avanzando la punta del endoscopio por el sitio de la estenosis que produce el vólvulo y posteriormente llevar la punta del endoscopio al duodeno, realizando un torque del endoscopio en el sentido de las manecillas del reloj, con lo que se logra la desvolvulación completa ${ }^{(14)}$. La maniobra tipo J o de retroversión, también se ha utilizado para realizar gastropexias.

El tratamiento laparoscópico tiene indicaciones tales como: reducir el vólvulo, anclar el fondo gástrico al diafragma, unir la curvatura mayor del estómago a la pared abdominal, además de realizar algunas reparaciones diafragmáticas. La técnica laparoscópica está indicada en pacientes portadores de vólvulos crónicos y en los que no existe necrosis o isquemia gástrica certificada. La gastrectomía total se realiza sólo en casos de necrosis gástrica.

La anatomía patológica cuenta con una gran pieza 
operatoria en los casos agudos en que intraoperatoriamente se detecta necrosis gástrica y ante la cual debe realizarse una gastrectomía que por lo general es total. Esta necrosis sobreviene a la isquemia que se genera por dos mecanismos, la obstrucción vascular propiamente tal y el perjuicio en la irrigación intramural por la dilatación aguda ${ }^{(15)}$.

Los hallazgos incluyen necrosis del espesor de la pared en el área gástrica más cercana al sitio de mayor obstrucción vascular, necrosis de la mucosa y la submucosa a mediana distancia del mismo sitio e infiltrado de origen inflamatorio en las áreas más periféricas. También se puede evidenciar microperforación transmural y signos de inflamación-necrosis de las estructuras vasculares (Figuras 12, 13).

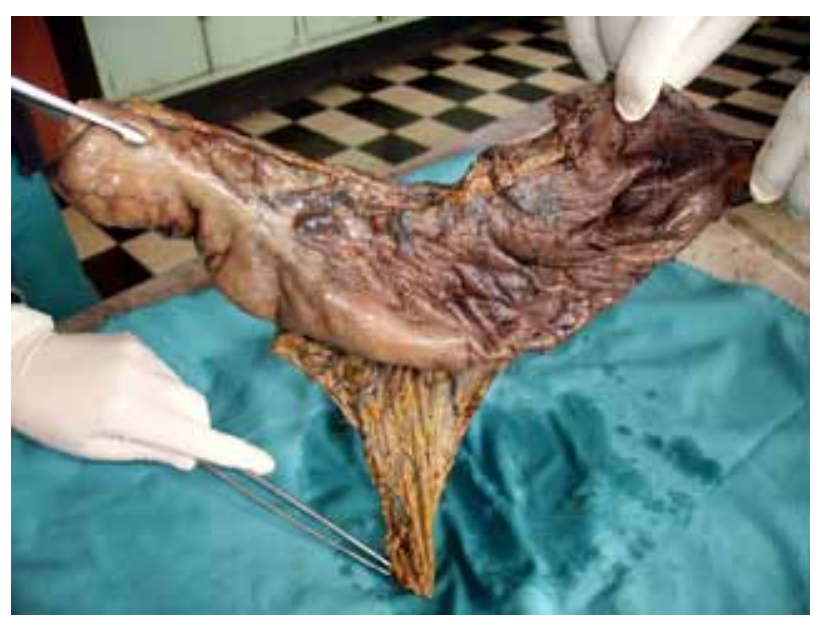

Figura 12. Pieza de anatomía patológica en que se observa compromiso vascular e isquémico de cuerpo y fondo gástrico

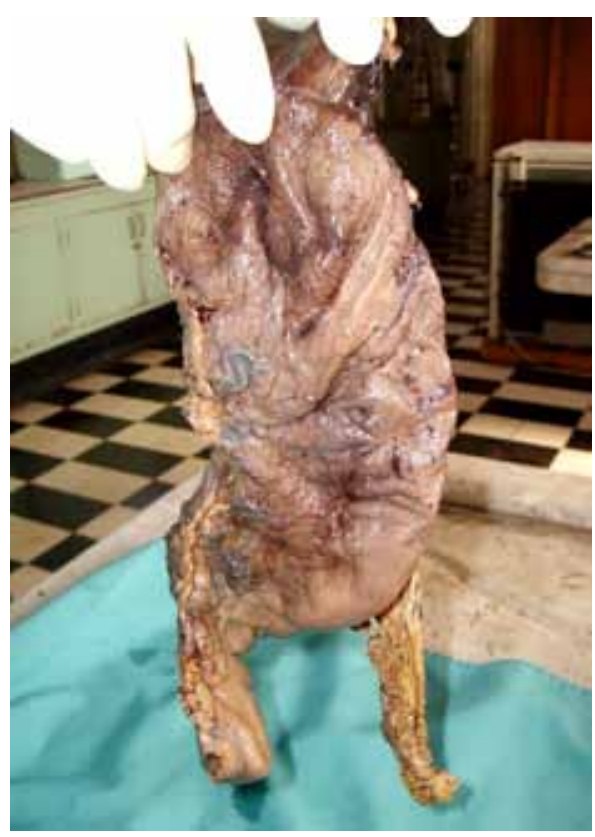

Figura 13. Pieza de anatomía patológica que demuestra perforación parietal macroscópica.
La mortalidad de la patología varía en las diferentes publicaciones dependiendo si se consideran en la estadística los vólvulos gástricos crónicos y el tipo de cirugía realizada, estando la mortalidad en un rango entre el 12 y el $50 \%{ }^{(16)}$, elevándose a más del $80 \%$ en los casos en que se encuentra aire portal.

\section{Conclusión}

El vólvulo gástrico es una entidad diagnóstica de baja frecuencia con discreta expresión en la literatura mundial, siendo la mayor parte de ellos reportes de casos. Existe un amplio espectro de patología que va desde la presentación crónica, subdiagnosticada y de sintomatología inespecífica hasta la entidad aguda con presentación clínica abrupta, de ominoso pronóstico y que puede llevar a la muerte del paciente.

Es el rol fundamental de los métodos diagnósticos radiológicos, orientar la presencia, mecanismo de ocurrencia y complicaciones asociadas con el objeto de diagnosticar tempranamente, planificar adecuadamente el enfrentamiento terapéutico y disminuir la morbimortalidad de esta patología.

\section{Agradecimientos}

Dr. Alejandro Readi V, Médico especialista en Cirugía Hospital del Salvador, Santiago de Chile

Dr. Pablo Villegas, Médico Anátomo-Patólogo Hospital del Salvador, Santiago de Chile

\section{Bibliografía}

1. Goretty Cabrera-Tovar M, Renedo-Ríos J, Tejeda-Tapia H. "Vólvulo gástrico. Informe de un caso", Acta Pediatr Mex 2009; 30(3): 163-166.

2. Gottlieb C, Lefferts D, Beranbaum S. "Gastric Volvulus, part I" Rad. Therapy \& Nuclear Med. 1954; 72: 609-615.

3. Darani A, Mendoza-Sagaon M, Reinberg O. "Gastric volvulus in children". J Pediatr Surg 2005; 40(5): 855858.

4. Sánchez Santacruz Y, Fernández Marín I. "Cartas al Director: Vólvulo gástrico como causa infrecuente de dolor abdominal". Rev. Esp. Enferm. Dig (Madrid) 2009; 101(7): 506-519.

5. Oh S, Han B, Levin T, Murphy R, Blitman N, Ramos C. Gastric volvulus in children: the twists and turns of an unusual entity. Pediatr Radiol 2008; 38: 297-304.

6. Payer A. Volvulus ventriculi und die Achsendrelung des Magens. Mitt Grenzab Med Chir 1997; 20: 686-694.

7. Peterson C MD, Anderson J MD, Hara A MD, Carenza J, Menias C. "Volvulus of the Gastrointestinal Tract: Appearances at Multimodality Imaging". RadioGraphics 2009; 29: 1281-1293.

8. Kotobi H, Auber F, Otta E, Meyer N, Audry G, Hélardot $P$. Acute mesenteroaxial gastric volvulus and congenital diaphragmatic hernia. Pediatr Surg Int 2005; 21: 674-676.

9. Matsuzaki Y, Asai M, Okura T, Tamura R. Ultrasonography of gastric volvulus. Intern Med. 2001; 40(1): 23-27.

10. Casella V, Avitable G, Segreto S, Mainenti P. CT findings in a mixed-type acute gastric volvulus. Emergency 
Radiol 2011.

11. Waselle JA, Norman J. Acute gastric volvulus: Pathogenesis, diagnosis, and treatment. Am J Gastroentero 1993; 88: 1780-1784.

12. Tanner NC. Chronic and recurrent volvulus of the stomach with late results of colonic displacement. Am J Surg. 1968; 115: 505-515.

13. Morelli U, Bravetti M, Ronza P, Cirochi R, De Sol A, Spizzirri A, et al. Laparoscopic anterior gastropexy for chronic recurrent gastric volvulus: a case report.
Journal of Medical Case Reports 2008; 2: 244.

14. Gómez Martín A, Ortiz C. Manejo endoscópico del vólvulo gástrico. Asociación colombiana de Gastroenterología, Endoscopía digestiva, Coloproctología y Hepatología. Febrero 2011.

15. Norese M, De Anton R, Sarotto L. Dilatación gástrica aguda complicada con necrosis. Rev Arg Cirugía 2006; 91(3-4): 105-107.

16. Koger KE, Stone JM. Laparoscopic reduction of acute gastric volvulus. Am Surg 1993; 59: 325-328. 